\title{
Melanoma immunotherapy
}

Editorial

\section{Abhishek D. Garg, Aleksandra M. Dudek-Peric and Patrizia Agostinis}

Cutaneous malignant melanoma is amongst the most aggressive cancer types, which typifies the paradox of being simultaneously highly antigenic and highly immunoevasive $[1,2]$. However, various anti-melanoma immunotherapies have shown limited clinical success e.g. IL2, IFN- $\alpha 2 \mathrm{a}$ or dendritic cells (DCs)-based vaccines [1-3]. Subsequent research showed that tumor-induced immunosuppression caused failure of above mentioned immunotherapies and thus overcoming this was proposed to be the key to success. This assumption proved correct, as evident by the impressive anti-melanoma clinical responses achieved with anti-CTLA4/PD-1/PD-L1 antibodies (i.e. immune-checkpoint inhibitors or ICIs) $[2,3]$. However, despite this success, there remains a sizeable subset of melanoma patients that don't respond to ICI-therapies [3]. While efforts are underway to dig out new immunological therapeutic targets yet recent research suggests that cancer cell-autonomous events can also play an important role in resistance to immunotherapies. For instance, recently melanoma cells were reported to mount resistance against anti-CTLA4 immunotherapy by up-regulating surface PD-L1 and thereby causing T cell exhaustion (Figure 1) [2]. Similarly, melanoma cellautonomous WNT/ $\beta$-catenin signaling was found to cause $\mathrm{T}$ cell exclusion from tumor microenvironment thereby creating ICI-therapy resistance (Figure 1) [3].

The above research on cancer cell-autonomous resistance has largely focused on ICI-therapies. However, it is also widely recognized that a durable "resetting" of the tumor microenvironment towards immune-susceptibility requires effective treatment with cytotoxic drugs that activate anticancer immunity [4-6]. We believe that the highest degree of such "reset" is achievable by inducers of immunogenic cell death (ICD) $[5,6]$. ICD is a cell death routine triggered by a limited set of assorted anticancer therapies (anthracyclines, radiotherapy, photodynamic therapy/PDT, oncolytic viruses), that is accompanied by danger signaling-driven, spatiotemporally defined, surface exposure or secretion/release of damage-associated molecular patterns (DAMPs) like surface-calreticulin (ecto-CRT, a quintessential 'eat me' signal that mediates immunogenicity) [4-6]. These DAMPs than interact with their cognate receptors, and help in instigating a potent anti-tumor immune response that helps eradicate residual cancer cells $[4,5]$. ICD has shown significant preclinical promise in a number of experimental models and some clinical promise in patients of lung cancer, breast cancer, ovarian cancer and lymphoma [4-6].

Remarkably, melanoma, which shows palpable preclinical susceptibility to ICD, has usually failed to show clinical responsiveness to single-agent ICD inducers like, anthracyclines/doxorubicin [7]. These paradoxical results have driven some of our recent research endeavors aimed at studying the ICD-melanoma link. We have found that melanoma cell-autonomous resistance to ICD can operate on two major levels i.e. macroautophagy activity $[6,8]$ (Figure 1) and general capability of surface-exposing CRT $[1,4]$ (Figure 1), as further discussed below.

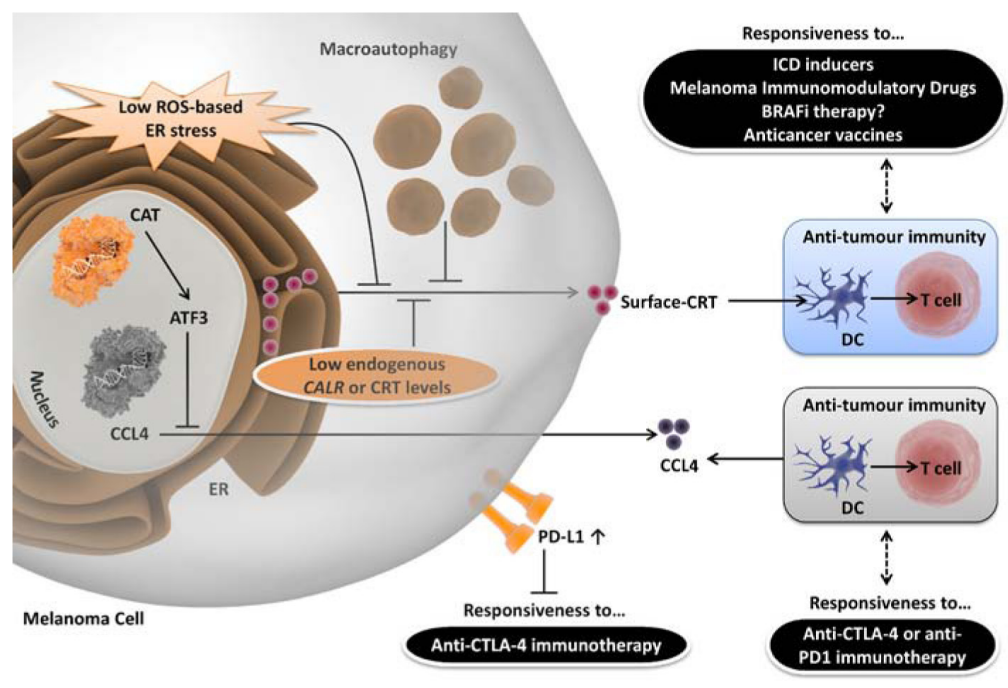

Figure 1: Schematic representation of melanoma cell-autonomous resistance mechanisms against immunotherapeutic paradigms. Melanoma cell-autonomous resistance can be posed to ICI-drugs (antiCTLA4/anti-PD1 immunotherapy) either via increased surface PD-L1 levels or through WNT/B-catenin (CAT) signalingbased increase in ATF3 levels, which in turn reduce the $\mathrm{T}$ cell-attracting chemokine, CCL4. On the other hand, resistance against the effect of ICD, vaccines or immunomodulatory chemotherapeutics/drugs (melphalan/BRAFi) can operate on the level of low surface-CRT. Low ROS-based ER stress, high macroautophagy and/ or low endogenous CRT/CALR levels can all contribute towards decreased surface-CRT. 


\section{Macroautophagy activity}

Using a bona fide ICD inducer, Hypericin-based PDT (Hyp-PDT) [6], we found that human melanoma cell-associated macroautophagy suppresses exposure of ecto-CRT (possibly by reducing oxidative-ER stress), which in turn reduces maturation of the interacting DCs, DC-derived IL6 production and proliferation of IFN- $\gamma$ producing $\mathrm{CD}^{+} / \mathrm{CD} 8^{+} \mathrm{T}$ cells (Figure 1) [6]. Thus, these results unraveled a role for ROS-induced autophagy in weakening the functional interaction between dying melanoma cells and immune cells [6]. These results were recently, partially, extended to BRAF ${ }^{\mathrm{V} 600 \mathrm{E}}$ inhibitorresistant melanoma cells where autophagy was shown to suppress exposure of ecto-CRT and ecto-HSP90 [8] (Figure 1). Nevertheless, it remains to be seen whether this activity of cell-associated autophagy also weakens the interactions between immune cells and $\mathrm{BRAF}^{\mathrm{V} 600 \mathrm{E}}$ inhibitor-resistant melanoma cells [8].

\section{General capability of surface-exposing CRT}

In a recent report we demonstrated the existence of a broad ICD-resistance mechanism using an AY27 rat bladder cancer model and two bona fide ICD inducers (mitoxantrone and Hyp-PDT) [4]. This ICD-resistant phenotype stemmed from low endogenous CRT protein levels in cancer cells (i.e. CRT ${ }^{\text {low }}$-phenotype) which resulted in defective ecto-CRT levels (Figure 1), which further caused severely reduced phagocytic clearance of treated cancer cells, which ultimately lead to the failure of tumor-rejecting immunity [4]. Interestingly, we found that a subset of cancer patients of various cancer-types tend to exhibit $C A L R^{\text {low }}$ or CRT ${ }^{\text {low }}$-tumors [4]. Moreover, we observed that tumoral $C A L R^{\text {high }}$-phenotype was predictive of positive clinical responses to therapy with ICD inducers like radiotherapy or paclitaxel in non-small cell lung or ovarian cancer patients, respectively (but not non-ICD inducer like topotecan in ovarian cancer) [4]. Additionally, tumoral CALR levels positively correlated with the levels of genes relevant for phagosome maturation or processing in only the clinical ICD set-up [4] (Figure 1). Importantly, we found that a subset of melanoma patients also had the tendency to show $\mathrm{CRT}^{\text {low }}$-tumors thereby hinting at the possible existence of above resistance mechanism in melanoma [4]. Possibility of such resistance mechanism in melanoma is of high implication since our research has found ecto-CRT to be crucial for immunogenicity of dying melanoma cells [1]. More specifically, we have shown that a well-established anti-melanoma chemotherapeutic, melphalan fails to induce sufficiently high immunogenicity in vivo, because it is not able to induce the relevant threshold levels of reactive oxygen species (ROS)-based ER stress required for ecto-CRT induction (Figure 1) [1]. Ecto-CRT on melphalan-treated melanoma cells was "restored" when the low ROSbased ER stress was increased by combining with the ER stressor, thapsigargin [1]. Importantly, we also observed that dying melanoma cells were largely reliant on ectoCRT for immunogenicity since ecto-HSP90, which was emphatically exposed by melphalan-treatment failed to mediate immunogenicity [1]. In near future, it would be interesting to find whether overall CRT or CALR levels in melanoma are predictive of clinical responses to ICD or immunotherapy and/or regulate overall levels/spatial distribution of $\mathrm{CD} 8^{+} \mathrm{T}$ cell-infiltrates.

In conclusion, there clearly exist melanoma cell-autonomous mechanisms that disrupt responses to immunotherapy with ICI-drugs or ICD-inducers. However, a future exome-sequencing or deep-sequencing study utilizing melanoma patient samples is required to characterize melanoma genotypes that associate with poor $\mathrm{T}$ cell infiltration and/or clinical responses to antimelanoma therapeutics.

\section{CONFLICT OF INTEREST}

No potential conflicts of interest were disclosed.

Abhishek D. Garg: Cell Death Research \& Therapy (CDRT) Lab, Department of Cellular Molecular Medicine, KU Leuven University of Leuven, Belgium

Correspondence: Abhishek D. Garg, email abhishek.garg@ med.kuleuven.be

Keywords: immunogenicity, immunogenic cell death, calreticulin, immune-checkpoint blockade, patient treatment

Received: July 28, 2015

Published: August 31, 2015

\section{REFERENCES}

1. Dudek-Peric AM, et al. Cancer Res. 2015; 75: 1603-1614.

2. Twyman-Saint Victor C, et al. Nature. 2015; 520: 373-377.

3. Spranger $\mathrm{S}$, et al. Nature. 2015; 523: 231-235.

4. Garg AD, et al. Oncotarget. 2015; PMID: 26314964. [Epub ahead of print].

5. Garg AD, et al. Oncoimmunology. 2015; DOI: 10.1080/2162402X.2015.1069938.

6. Garg AD, et al. Autophagy. 2013; 9: 1292-1307.

7. Smylie MG, et al. Invest New Drugs. 2007; 25: 155-159.

8. Martin S, et al. Biochem Pharmacol. 2015; 93: 290-304 